\title{
Three-Dimensional Simulation of Intrinsic Stress Build-Up in Thin Films
}

\author{
H. Ceric, C. Hollauer, and S. Selberherr \\ Institute for Microelectronics, TU Wien, Gußhausstraße 27-29/E360, 1040 Wien, Austria \\ Phone: +43-1-58801/36032, Fax: +43-1-58801/36099, E-mail: ceric@iue.tuwien.ac.at
}

\begin{abstract}
We present a method for prediction of stress in poly-SiGe thin films based on the texture evolution. Models for different stress generation mechanisms are discussed and integrated in an overall simulation scheme. As example a three-dimensional cantilever structure is used to demonstrate the introduced approach, and simulation results are successfully compared with experiment.
\end{abstract}

\section{INTRODUCTION}

Thin film deposition is a widely used technique for the fabrication of MEMS (Micro-Electro-Mechanical Systems) devices. These technique is required to establish free-standing structures which can induce or sense a mechanical movement. During the thin film deposition process and aftermath an intrinsic stress is generated. In subsequent process steps, a stressed layer, usually an important component part of the desired MEMS device, is left free-standing. As a consequence the process induced stress can relax and deform the layer in an undesirable way.

Poly-SiGe has been promoted as a material suitable as structural layer for several MEMS applications [1]. It possesses good mechanical and electrical properties which can be obtained at much lower temperatures $\left(400{ }^{\circ} \mathrm{C}\right)$ as compared with polysilicon $\left(800{ }^{\circ} \mathrm{C}\right.$ and higher).

Different aspects of the connection between microstructure and stress have been investigated in the last 30 years. The focus was mostly on some specific grain-grain boundary configurations in early or mature stages of microstructure evolution [2]. As a result there are numerous models derived on the basis of continuum mechanics, which are applicable only for highly simplified situations. On the other side a group of researchers, mostly mathematicians, has developed complex models for describing morphology of the microstructural evolution, a development which culminates in multi-level set models of grain evolution [3], [4]. Such models can to a high degree reproduce the realistic grain boundary network, but they do not include stress [4]. The goal of this work is the integration of microstructure models which describe strain development due to grain dynamics with the macroscopic mechanical problem. The solution of the mechanical problem provides a distribution of the mechanical stress in tensor form, which can further be used to assess mechanical stability of MEMS structures.

\section{SOURCES OF INTRINSIC STRESSES}

For the investigation of stress effects during deposition we focus on the low pressure chemical vapor deposition (LPCVD) of $\mathrm{SiGe}$ on the silicon-dioxide substrate, which is a widely used technique for MEMS application.

In the first phase of the deposition process, islands with varying crystal orientation are formed and grow isotropically. The radial growth of the material islands mainly depends on the Si-Ge ratio, the substrate temperature, and the silane $\left(\mathrm{SiH}_{4}\right)$ and germane $\left(\mathrm{GeH}_{4}\right)$ flow density. In the course of further deposition these islands start to coalescence, which forces the islands to grow in the height instead in a direction parallel to the substrate surface. The islands are subsequently transformed from an island shape to a grain-like shape. The orientation of the crystal structure in a single grain (e.g. perpendicular to the substrate surface) is independent of the neighboring grains, since due to the amorphous substrate, it is not possible to evolve a perfect crystal structure in the first atom layers [5]. Another aspect is that the deposition takes place at elevated temperatures. When the temperature decreases to room temperature, the volumes of the grains decrease and the stresses at the grain boundaries increase.

Intrinsic stresses observed in thin metal films are generally tensile [2], the main sources of these stresses are presented in the following sections.

\section{A. Coalescence of the Grain Boundaries}

In the early stage of the film growth, coalescence of the small grains generates a tensile stress. The closing of the gaps between crystallites causes their elastic deformation. The driving force of this phenomenon is cohesion [6].

\section{B. Misfit Stresses}

These stresses arise when the crystal lattice of the thin film and substrate are forced to line up perfectly. The influence of these stresses is significant in the initial phase of the thin film deposition [7].

\section{Rearrangement of the Atoms}

Metal is deposited in non-equilibrium state, subsequent atom rearrangements tend to reach equilibrium. Generally such rearrangements result in the shrinkage of the film. While the film is attached to the substrate a tensile stress develops [8].

\section{Grain Growth}

Grain boundaries are less dense than the grain lattice. Therefore, elimination of grain boundaries leads to a densification of the film and buildup of the tensile stress [9]. 


\section{E. Annihilation of Excess Vacancies}

The annihilation and the dynamics of the crystal vacancies produce a local volume which leads to stresses in the film when attached to the substrate. The vacancies annihilate in the grains, at the grain boundaries, at the metal free surface, and at the surface of the internal cavities. In the case of vacancy annihilation at the metal free surface and at internal cavities' surfaces no stress is produced, but vacancy annihilation at grain boundaries causes motion of the crystals toward each other which would result in a planar contraction of the film, if it were not attached to the substrate. But since the substrate prevents contraction, biaxial tensile stress is built instead [2].

\section{F. Thermal Stress}

This stress is caused by the thermal mismatch between the metal film and the substrate. It develops during cooling down to room temperature.

\section{Physical Modeling}

Different process conditions and underlying physical phenomena make it convenient to describe the previously described sources of stress in initial mode and transient mode microstructure models. The initial mode of the thin film growth model considers development of the first grain layer (Figure 1). The dominant stress components in this case are caused by coalescence of the grain boundaries and misfit stresses. As transient mode we consider a situation where more than one grain layer is deposited (Figure 2). The stresses developed in the initial mode are now present as the residual stresses. The stress build-up in the transient mode is caused by the grain growth and non-equilibrium vacancy dynamics.

1) Stress-Strain Model: The transient evolution of the displacement vector $\mathbf{u}=\left(u_{1}, u_{2}, u_{3}\right)$ is determined by Newton's law,

$$
\rho_{\mathrm{m}} \frac{\partial^{2} u_{i}}{\partial t^{2}}=\frac{\partial \sigma_{i, j}}{\partial x_{j}}
$$

where $\rho_{\mathrm{m}}$ is the density of the metal. Since accelerations are small during the thin film deposition, we make the assumption,

$$
\frac{\partial \sigma_{i, j}}{\partial x_{j}}=0
$$

The standard relationship between the total strain tensor and the components of displacement $\mathbf{u}$ reads

$$
\varepsilon_{i j}=\frac{1}{2}\left(\frac{\partial u_{i}}{\partial x_{j}}+\frac{\partial u_{j}}{\partial x_{i}}\right) .
$$

The stress tensor $\sigma_{i j}$ is in the case of the film growth time dependent, and generally consists of the elastic $\sigma_{i j}^{\text {el }}$ and inelastic component $\sigma_{i j}^{\text {in }}$, i.e.,

$$
\sigma_{i j}=\sigma_{i j}^{\mathrm{in}}+\sigma_{i j}^{\mathrm{el}} .
$$

The elastic component for the isotropic materials is given by Hook's law

$$
\sigma_{i j}^{\mathrm{el}}=\lambda \varepsilon_{k k} \delta_{i j}+2 \mu \varepsilon_{i j}
$$

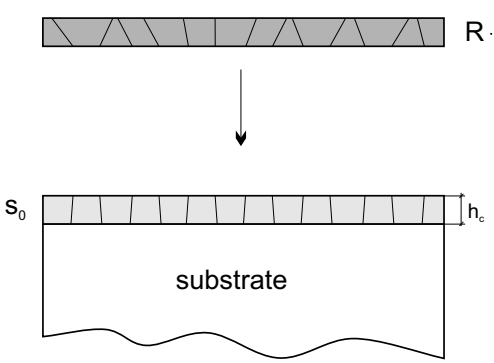

Figure 1: Initial mode. $R$ is the deposition rate and $h_{c}$ is the film thickness immediate after grain formation.

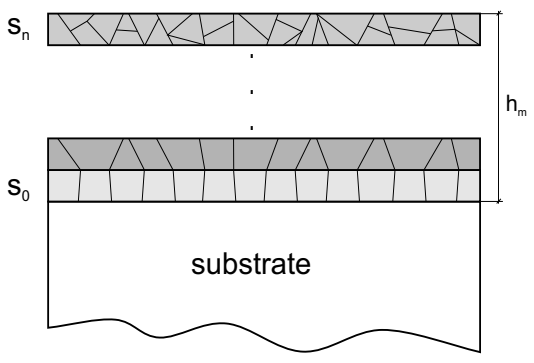

Figure 2: Grains formed in the initial mode continue development in transient mode.

where $\lambda$ and $\mu$ are the Lame coefficients. Below we will discuss the composition of this inelastic stress component in detail. From (2)-(5), the main equilibrium condition due to all stress components is

$$
\frac{1}{1+\nu} \nabla^{2} u_{i}+\frac{1}{1-2 \nu} \frac{\partial}{\partial x_{i}}(\nabla \cdot \mathbf{u})=-\frac{2}{E} \frac{\partial \sigma_{i j}^{i n}}{\partial x_{i}},
$$

where $E$ is the Young modulus and $\nu$ the Poisson ratio. The right side of equation (6) represents the influence of the residual stress load.

2) Initial Mode: The initial mode of the thin film growth model considers development of the first grain layer (Figure 1). The dominant stress components in this case are caused by coalescence of the grain boundaries and misfit stresses.

Assuming a spherical grain shape at the initial stage of the growth, the average stresses $\sigma_{x x}^{i n}, \sigma_{y y}^{i n}$, and $\sigma_{z z}^{i n}$ in the film of thickness $h_{c}$ are [10]

$$
\sigma_{x x}^{i n}=\sigma_{y y}^{\text {in }}=\frac{2 \pi \gamma}{3 h_{c}}, \quad \sigma_{z z}^{i n}=0,
$$

where $\gamma$ is the surface energy of the contacting spheres. Misfit stresses occur in crystalline films due to geometric mismatch at interface boundaries between crystalline lattices of films and substrates. The film substrate interface is characterized by the misfit parameter [11]

$$
f=2 \frac{a_{f}-a_{s}}{a_{f}+a_{s}},
$$

where $a_{f}$ and $a_{s}$ are the lattice parameters of the film and substrate, respectively. The nonzero components of the misfit stress tensor are 


$$
\sigma_{x x}^{\text {in }}=\sigma_{y y}^{\text {in }}=\frac{E f}{1-\nu^{2}}, \quad \sigma_{z z}^{i n}=\nu \sigma_{x x}^{\text {in }}=\nu \sigma_{y y}^{\text {in }} .
$$

3) Transient Mode: As transient mode we consider a situation where more than one grain layer is deposited (Figure 2). The stresses developed in the initial mode are now present as residual stresses. The stress buildup in the transient mode is caused by grain growth and non-equilibrium vacancy dynamics. The inelastic stress component of the transient mode is given by

$$
\begin{aligned}
\sigma_{x x}^{\text {in }}=\sigma_{y y}^{\text {in }}= & \sigma_{z z}^{\text {in }}=-\frac{2 E}{1-\nu}\left(\frac{1}{L}-\frac{1}{L_{0}}\right) \Delta a+ \\
& +\frac{4 E \Omega}{L(1-\nu)} \sqrt{\frac{D_{V} t}{\pi}}\left(C_{\text {bulk }}-C_{\mathrm{gb}}\right) .
\end{aligned}
$$

The first term on right side of this equation represents stresses developed due to grain growth [9]. $L$ is the current grain diameter and $L_{0}$ one inherited from the initial mode. $\Delta a$ is the excess volume per unit area of grain boundary, which has a value in the order of an atomic diameter. The second term of (10) is related to the annihilation of vacancies at the grain boundaries. The annihilation sites are transformed into the gaps between the grains, which are closed by straining of the grains. $D_{V}$ is the bulk vacancy diffusivity, $C_{\text {bulk }}$ is the vacancy concentration inside the grain, and $C_{\mathrm{gb}}$ in the grain boundary respectively.

The dynamics of the grain growth is given by [9]

$$
\frac{\partial L}{\partial t}=\frac{D^{*} \Omega}{k_{B} T \delta} \Delta p
$$

where $\Omega$ is the atomic volume, $\delta$ is the average jump distance in the grain boundary, $k_{B} T$ is the thermal energy, and $p$ is the hydrostatic pressure calculated as the trace of the stress tensor,

$$
p=-\frac{1}{3} \sigma_{i i}
$$

$D^{*}$ is the effective diffusivity [5]

$$
D^{*}=\delta^{2} \alpha \exp \left(-\frac{\Delta H}{k_{B} T}\right)
$$

where $\alpha$ is the atomic frequency and $\Delta H$ is the activation energy of the boundary migration. We assume that due to the material deposition the film thickness is growing with the rate $R$ in $x_{3}$-direction during the time $t_{D}$. The tensor stress distribution function $\sigma^{D}\left(x_{1}, x_{2}, x_{3}\right)$ at the end of the thin film deposition process is calculated as

$$
\sigma_{i j}^{D}(\mathbf{x})=\int_{0}^{t_{D}}\left(\frac{\partial \sigma_{i j}(\mathbf{x}, t)}{\partial x_{3}} R+\frac{\partial \sigma_{i j}(\mathbf{x}, t)}{\partial t}\right) d t
$$

where $\sigma_{i j}(\mathbf{x}, t)=\sigma_{i j}\left(x_{1}, x_{2}, x_{3}, t\right)$ fulfill equations (6)-(13).
4) Cooling Down to Room Temperature: The developed inelastic stress component due to thermal mismatch of the film and the substrate material is given by,

$$
\sigma_{x x}^{\mathrm{in}}=\sigma_{y y}^{\mathrm{in}}=\sigma_{z z}^{\mathrm{in}}=B \alpha\left(T-T_{0}\right) \delta_{i j},
$$

where $B=(3 \lambda+2 \mu) / 3$ is the bulk modulus, $\alpha$ is the thermal expansion coefficient, and $T_{0}$ is the room temperature.

\section{A. Simulation Approach}

There are basically two possibilities to numerically handle the models described in the previous section. The first one is continuum modeling of the polycrystalline domain and the second one is a multi phase level set approach.

1) Continuum Modeling: The stress problem is solved in the initial mode for a rectangular film geometry of thickness $h_{c}$. The only boundary condition used is zero displacement (i.e., $u_{i}(\mathbf{x})=0$ ) at the thin film/substrate interface. If we assume a rectangular simulation domain $S_{n}$ with the thickness $h_{n}>h_{c}$ for the discrete time $t_{n}$, which is loaded with the residual stress distribution $\sigma\left(\mathbf{x}, t_{n}\right)$, the stress distribution for the next time step $t_{n+1}$ is calculated by adding the relaxed layer of the thickness $R \cdot \Delta t$ on the top of the domain $S_{n}$, thus obtaining domain $S_{n+1}$ with thickness $t_{n+1}=t_{n}+R$. $\Delta t$. Additionally, the stress problem described by equations (6)-(14) is solved for the domain $S_{n+1}$ and a new stress distribution $\sigma\left(\mathbf{x}, t_{n+1}\right)$ is obtained. This calculation procedure is carried out until the final film thickness $h_{D}$ is reached.

2) Multi Level Set: Based on the approach presented in [3] and its improvement [4] it is possible to model polycrystalline kinetics of thin film grains by means of the multi phase level set method.

Assuming $N_{c}$ crystal seeds we assign different phase functions $\Phi_{l}, l=1, \ldots, N_{c}$ to each crystal. The governing equation for the evolution of each phase is

$$
\frac{\partial \Phi_{l}}{\partial t}+\eta \mathbf{v}_{l} \nabla \Phi_{l}=0
$$

where $\mathbf{v}_{l}$ are the corresponding velocity fields and $\eta$ is the function controlling the collision of the grains (phases).

In the original works [3], [4] the velocities of the evolving surfaces are related only to the surface energy of the evolving grains, however, it is possible to expand this connection by equations (11) and (12) thus including local stress effects.

Compared with continuum stress modeling, the application of the multi phase level set method is computationally more demanding, but it enables the simulation of some additional stress contributions like misfit inter-grain stress which develops due to the difference of neighboring crystal orientations and influence of the cavities at the grain boundaries.

\section{Simulation Results AND Discussion}

The approach developed in this work is applied to the experimental setting presented in [12]. The continuum modeling method (Section III-A.1) has been implemented for the simulation of microstructural stress evolution. Our simulation tool is capable of solving multi-physics equations by means of 


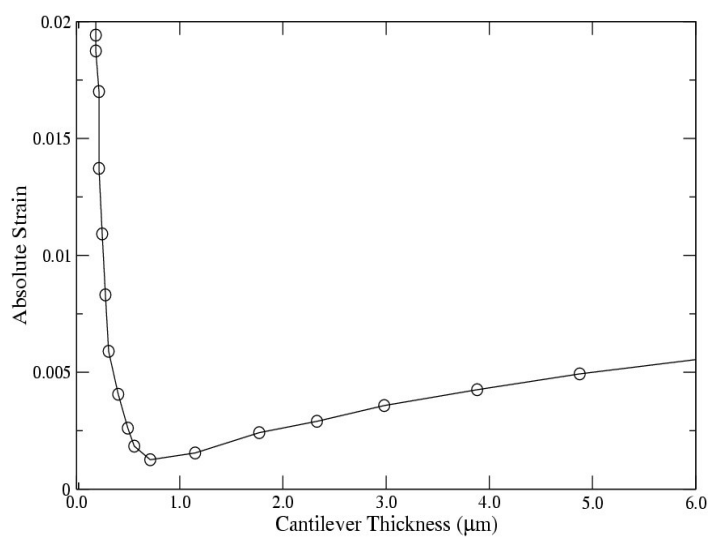

Figure 3: The run of strain curve through the thickness of thin fi $1 \mathrm{~m}$.

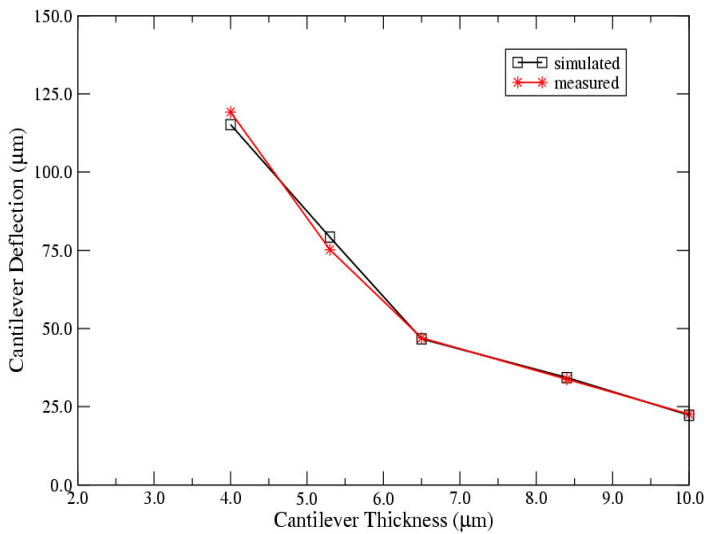

Figure 4: Comparison between the measured and the simulated cantilever deffections for different thicknesses.

the finite element method. All simulations have been carried out on a high performance IBM-AIX cluster. An intrinsic strain curve (Figure 3), which qualitatively is predicted by the model, was calibrated according to measurement results. Simulated cantilever deflections show good agreement with experimentally determined deflections (Figure 4). Figures 5 and 6 display results of three-dimensional cantilever deflection simulations. The distribution of the peak stress values is displayed by the red areas. After removing of middle layer, the cantilever is released (Figure 6). The reduction of high stress areas indicates a stress relaxation in the free standing cantilever.

\section{CONCLUSION}

We presented a simulation concept which connects microstructural mechanical properties of copper films to the overall stress distribution. The underlying model is designed by combining several earlier models which describe different microstructural contributions to stress build up. Simulation results are successfully compared with a experiment.

\section{ACKNOWLEDGMENT}

This work has been supported by the European Community with project PROMENADE, IST-2002-2.3.1.2.

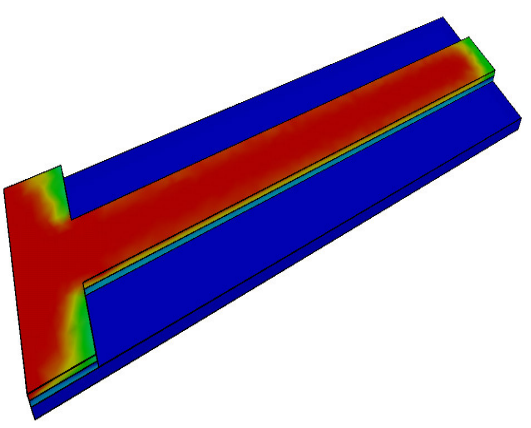

Figure 5: Stress distribution for fi xed cantilever.

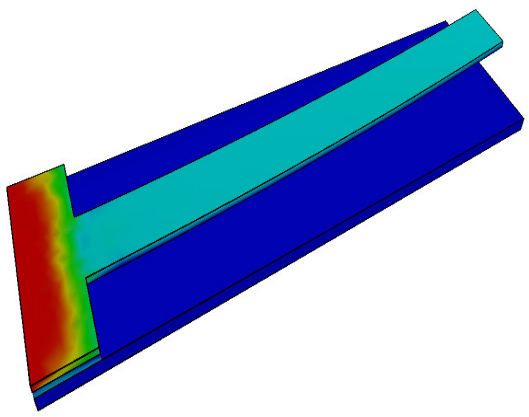

Figure 6: The build-up of intrinsic stresses induces cantilever deffection.

\section{REFERENCES}

[1] A. Witvrouw, M. Gromova, A. Mechta, S. Sedky, P. De Moor, K. Baert, and C. Van Hoof, Proceedings of the MRS Fall Meeting, Boston, MA, USA,1-5 December 2003.

[2] M. F. Dorner and W. D. Nix, CRC Critical Rev. Solid State Mater. Sci., vol.14, no.3, pp.225-267, 1988.

[3] G. Russo and P. Smereka, SIAM J. Sci.Comp, vol.21, no.6, pp.2073-2095, 2000.

[4] P. Smereka, X. Li, G. Russo, Srolovitz, SIAM J. Sci. Comp, vol. 53, pp.1191-1204, 2005.

[5] P. G. Shewmon, Transformations in Metals, McGraw-Hill, New York, 1969.

[6] R. W. Hoffman, Thin Solid Films, vol. 34, pp. 185-190, 1976.

[7] K. Cholevas, N. Liosatos, A. E. Romanov, M. Zaiser and E. C. Aifantis, Phys. Stat. Sol., vol. (b) 209, pp. 295-304, 1998.

[8] E. Klokholm and B. S. Berry, J. Electrochem. Soc., vol.115, no. 8, pp.823-826, 1968.

[9] P. Chaudhari, J. Vac. Sci. Techn., vol. 9, no. 1, 1972.

[10] L. B. Freund and E. Chason, J. Appl. Phy., vol. 89, pp. 4866-4873, 2001.

[11] S. V. Bobylev and I. A. Ovidko., Phys. Sol. Stat., vol. 45, no. 10, pp.1926-1931, 2003.

[12] A. Molfese, A. Mehta, and A. Witvrouw, Sensors and Actuators A, vol. 118, no. 2., pp. 313-321, 2005. 